Revue des patrimoines

\title{
Théorie et pratique du fruitier. Quelques données franciliennes : fouille, ethnographie et archives
}

Jean-Yves Dufour, Thalie Courelis et Jean-Jacques Péru

\section{(2) OpenEdition}

Journals

Édition électronique

URL : http://journals.openedition.org/insitu/10013

DOI : 10.4000/insitu. 10013

ISSN : 1630-7305

Éditeur

Ministère de la culture

Référence électronique

Jean-Yves Dufour, Thalie Courelis et Jean-Jacques Péru, «Théorie et pratique du fruitier. Quelques données franciliennes : fouille, ethnographie et archives », In Situ [En ligne], 21 | 2013, mis en ligne le 07 avril 2014, consulté le 22 avril 2019. URL : http://journals.openedition.org/insitu/10013 ; DOI : 10.4000/insitu.10013

Ce document a été généré automatiquement le 22 avril 2019

\section{(1) $9 \odot$}

In Situ Revues des patrimoines est mis à disposition selon les termes de la licence Creative Commons Attribution - Pas d'Utilisation Commerciale - Pas de Modification 4.0 International. 


\title{
Théorie et pratique du fruitier. Quelques données franciliennes : fouille, ethnographie et archives
}

\author{
Jean-Yves Dufour, Thalie Courelis et Jean-Jacques Péru
}

\section{Le témoignage de la littérature agronomique (Jean- Yves Dufour) ${ }^{12}$}

1 Tab. 1 - Le témoignage de la littérature agronomique sur les fruitiers. Phot. Dufour, JeanYves. ( Inrap 2010. Voir le document en annexe.

2 La lecture de 28 manuels d'agriculture (tab. 1) nous livre des informations techniques sur la connaissance des auteurs anciens sur les modes de conservation des fruits en fruitier. En dehors de Varron utilisé pour rappeler l'ancienneté de la conservation des fruits en lieu clos et distinct ces ouvrages échelonnés entre 1661 et 1900 couvrent largement la période qui nous intéresse. Qu'ils soient le fait de compilateurs (La Rivière (de) \& Du Moulin, 1739 ; Aubert la Chesnaye des Bois (de), 1751, Diderot et d'Alembert, 1782,...), de généralistes (Bona, 1873 ; Neufchâteau (de), 1828...) d'architecte (D'Aviler, 1755) ou des meilleurs spécialistes (La Quintinie, 1739; Bretonnerie, 1784; Mozard, 1814 ; Du Breuil, $1846 . .$.$) , ces manuels rendent compte des connaissances acquises et diffusées parmi un$ large éventail de lecteurs, puisqu'ils entrent aussi bien dans la catégorie des encyclopédies que dans celle des ouvrages pour dames (Millet-Robinet, 1893) ou des ouvrages didactiques (Martin et Roy, 1900). Nous ne prétendons certes pas les avoir tout consultés, mais certainement un éventail représentatif des conceptions sur le sujet.

3 Archéologue, nous avons procédé à une lecture orientée de ces manuels, c'est-à-dire que nous en avons extrait les informations nécessaires à l'identification du fruitier sur le terrain. L'emplacement du fruitier, son atmosphère, ses ouvertures, son exposition, l'épaisseur de ses murs et l'aménagement intérieur sont les sujets les plus abordés, car techniquement essentiels. Depuis Varron, la garniture des murs par des tablettes fait quasiment l'unanimité. Seule La Maison rustique du XIX ${ }^{\mathrm{e}}$ siècle (Brisseau de Mirbel, 1834) 
minimise volontairement le fruitier aménagé au profit de l'ensilage des fruits dans des fosses souterraines, technique plus couramment usitée pour les légumes. Cette technique n'est pas aussi expérimentale qu'elle en a l'air, Columelle et Pline recommandant également d'enterrer les grenades dans des fosses.

Les auteurs ont bien compris que la conservation des fruits dépend de la qualité de l'air et $\mathrm{du}$ «lieu ». On tente de contrôler l'atmosphère par divers critères de construction du fruitier. L'emplacement de la fruiterie est un sujet très débattu par les agronomes. Elle doit être éloignée de tout ce qui corrompt l'air, à savoir les fromages, les foins, les pailles, les fumiers, les latrines, les eaux stagnantes, le linge sale, les écuries... (11 auteurs, toutes périodes). Jusqu'à la décennie 1780 , les auteurs anciens recommandent de placer la fruiterie au rez-de-chaussée (8 mentions), voire à l'étage (3 mentions). Dans une brillante copie des uns sur les autres, huit auteurs du XVIII siècle répètent que «la cave ni le grenier ne sont pas propres pour faire une fruiterie ; la cave, à cause d'un goût de moisi \& d'une chaleur humide qui pourrit tout ; \& le grenier, à cause du froid et de l'humidité qui y pénètre la couverture. » (LIGER. La nouvelle maison rustique, II, 1777, p. 192).

Deux auteurs conviennent pourtant qu'il faut transporter les fruits dans la cave pendant le grand froid. À partir de La Bretonnerie (1784), la situation s'inverse : la cave saine constitue le meilleur fruitier pour 11 auteurs. L'abbé Rozier abrège le débat en expliquant qu'il importe peu que le fruitier soit dans une cave, au rez-de-chaussée ou à l'étage, tant que le lieu choisi est sec, ce qui est précisé par 10 auteurs.

6 Le cellier devient également recommandable pour 9 auteurs à partir de la décennie 1780 . Des murs épais (deux à quatre pieds), impénétrables à la gelée, sont mentionnés par 10 auteurs. L'exposition du fruitier influe naturellement sur sa température ; 17 auteurs en parlent. L'exposition au midi est la plus évoquée (13 mentions), suivie de celle au levant (10 mentions), de l'ouest (4 mentions) et du nord ( 3 mentions). Après le premier quart du XIXe siècle l'exposition occupe moins les agronomes qui n'abordent plus le sujet, sauf pour contredire des auteurs antérieurs (Paquet, 1844). Comme l'écrit Du Breuil, l'exposition devient un critère insignifiant dans le bon choix du fruitier.

7 Les doubles portes mentionnées par 16 auteurs sont destinées à protéger des variations atmosphériques. Les fenêtres donnent de la lumière naturelle mais posent la délicate question de la ventilation. Dès le milieu du XVII ${ }^{e}$ siècle (Bonnefons, 1661) et dans la période charnière des années 1780 , trois auteurs des plus avisés (La Bretonnerie, l'abbé Rozier et Parmentier) limitent au minimum le renouvellement de l'air, ne mentionnant donc pas de fenêtres et modérant le nombre de portes. À partir du milieu du XIX siècle, les auteurs sont indécis sur la question de l'air et de l'humidité. Au point que Paquet soutient que l'emplacement du fruitier dépend de la nature du sol des arbres fruitiers et du climat au moment de la cueillette des fruits ${ }^{3}$. On ne parle également plus de fenêtres à partir du milieu du XIX'e siècle, mais bien d'empêcher l'action néfaste de la lumière.

Les connaissances biologiques ne sont pas suffisamment approfondies durant les XVIII et $\mathrm{XIX}^{\mathrm{e}}$ siècles pour comprendre le pourrissement des fruits. Ce n'est qu'à partir de la fin du $\mathrm{XIX}^{\mathrm{e}}$ siècle que le développement de ces études permet de savoir que trois opérations sont nécessaires à la bonne conservation des fruits : si un local froid et ventilé évacue bien la chaleur et les gaz dégagés par la respiration du fruit (situation empiriquement reconnue par les anciens) une forte humidité (voisine de $90 \%$ ) est indispensable pour empêcher ou atténuer le flétrissement des fruits consécutif à leur transpiration ${ }^{4}$. 


\section{Un fruitier fouillé dans le château de Roissy-en-France, Val-d'Oise}

Figure 1
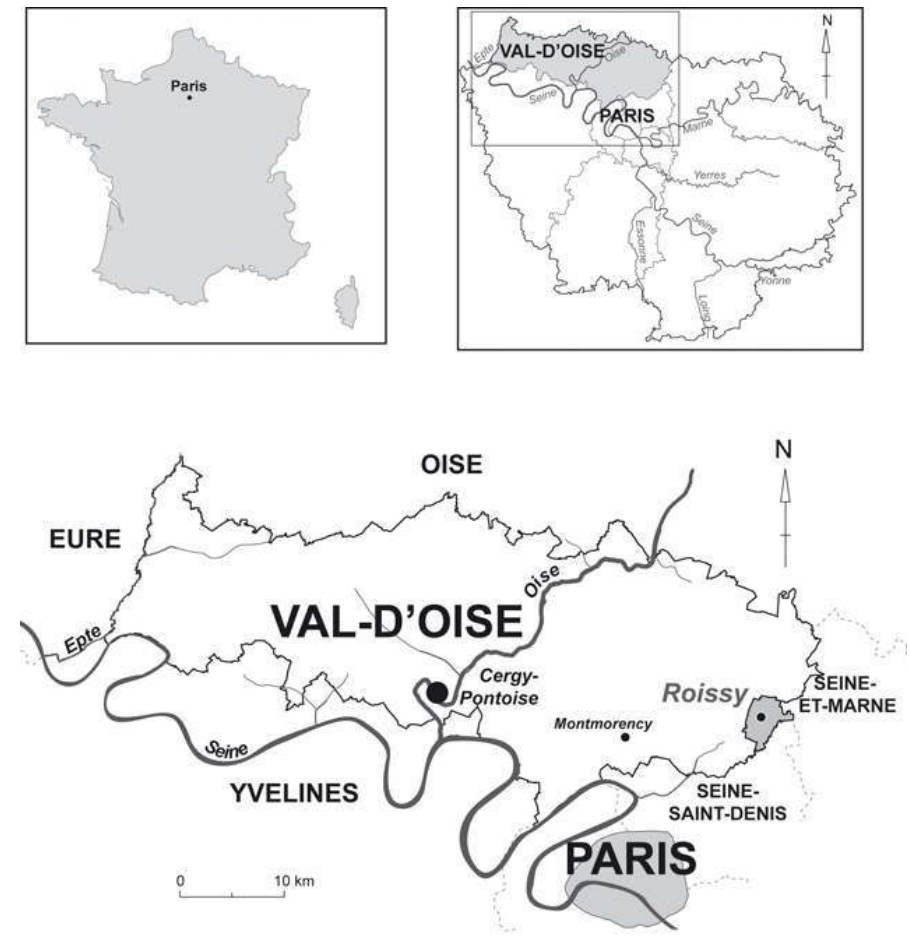

Fig. 1 : localisation de Roissy-en-France et de Montreuil.

Localisation de Roissy-en-France et de Montreuil.

Phot. Dufour, Jean-Yves, Belarbi, Mehdi. (C) Inrap 2010.

9 (fig. $\left.\mathbf{n}^{\mathbf{0}} \mathbf{1}\right)$

10 Le château de Roissy-en-France, dont le corps de logis a fait l'objet d'une fouille intégrale en 1999, évolue du XII ${ }^{\mathrm{e}}$ au XX ${ }^{\mathrm{e}}$ siècle. Le niveau des offices souterrains de l'époque moderne est partagé en de nombreuses pièces. Les caves du pavillon nord sont reliées aux offices par une longue galerie souterraine de 4,5 pieds de large (fig. $\mathbf{n}^{\circ} \mathbf{2}$ ). 


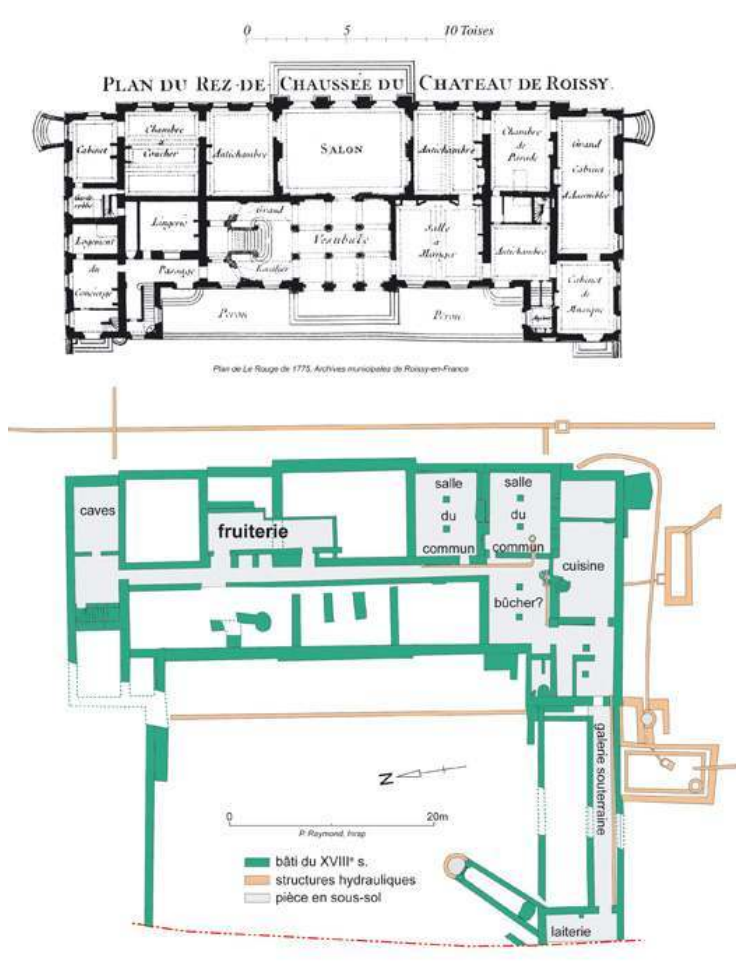

Fig. 2 : localisation de la fruiterie sur le plan archéologique du château de Roissy.

Localisation de la fruiterie sur le plan archéologique du château de Roissy.

Phot. Dufour, Jean-Yves, Belarbi, Mehdi. (c) Inrap 2010.

\section{Description des vestiges}

Une partie dut Le fait archéologique 1670 est composé de deux scellements au sol, espacés de $66 \mathrm{~cm}$ (2 pieds) pour des montants verticaux en bois de 11 x 7 à $8 \mathrm{~cm}$, avec feuillure signalant l'ouverture d'une porte sur la galerie. L'accès à la pièce souterraine étudiée se fait par un escalier (F.1143) positionné dans l'angle nord-ouest. Une seconde ouverture est visible dans la partie sud-ouest. Quel intérêt trouvent les constructeurs, à desservir une cave par deux portes ${ }^{5}$ ? Au XVIII ${ }^{\mathrm{e}}$ siècle, un seuil ${ }^{6}$ est construit devant l'interruption du mur ${ }^{7}$ qui sert de passage. La même maçonnerie ${ }^{8}$ prend place sous le placard. Positionnée à l'intérieur du placard, il est difficile d'y voir un seuil. La cave (ré)utilisée mesure 12,1 $\mathrm{m} \mathrm{x}$ 2,8 à 3,6 m, soit $37,9 \mathrm{~m}^{2}$ ou 10 toises en carré. Vingt-cinq traces de scellements ont été relevées sur le sol et un peu moins sur les murs. Elles semblent indiquer la présence de constructions de bois ; placards, étagères, consoles. Dans le sol, contre le mur oriental, la fouille a révélé une petite cuve (F. 1591) circulaire (diamètre $35 \mathrm{~cm}$ ) à parois verticales et fond plat atteint dès $30 \mathrm{~cm}$ de profondeur. Cette «cuve » est recouverte d'un plâtre fin gris clair sur $1,5 \mathrm{~cm}$ d'épaisseur. 
Figure 3

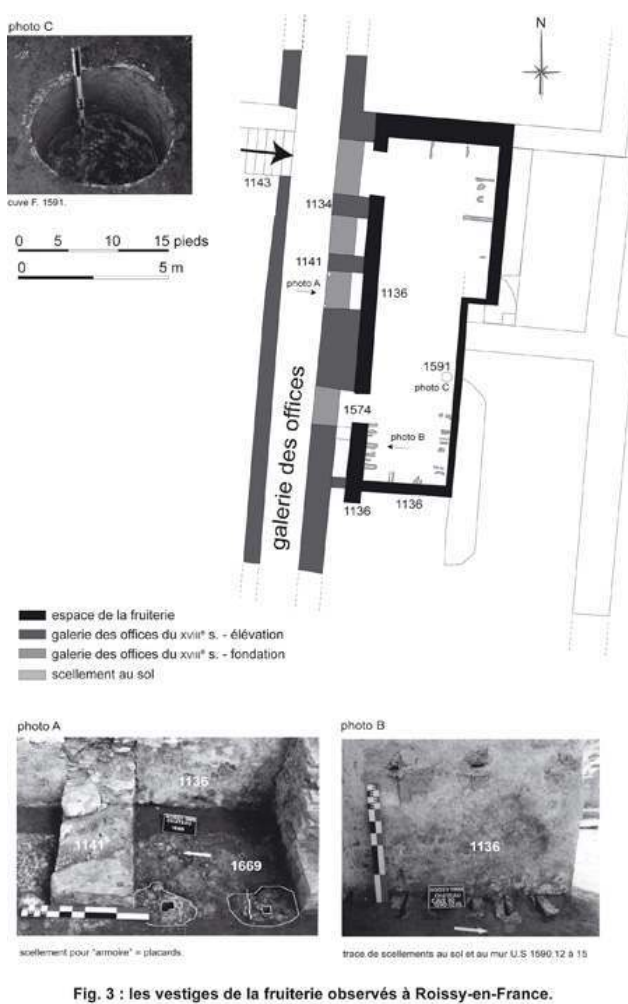

Les vestiges de la fruiterie observés à Roissy-en-France.

Phot. Dufour, Jean-Yves, Belarbi, Mehdi. (c) Inrap 2010.

\section{Interprétation : une fruiterie}

13 La lecture archéologique des manuels d'agriculture anciens nous permet de bien interpréter ces vestiges et de définir sans hésitation cette pièce comme une fruiterie. La pièce est bien isolée dans les caves ; cette localisation décriée par les agronomes pendant une grande partie du XVIII ${ }^{\mathrm{e}}$ siècle, est reconnue comme un emplacement sain à partir de la décennie 1780. La localisation en sous-sol du fruitier semble correspondre à une partie de la réalité, comme en témoigne également le plan du château de Champs-sur-Marne ${ }^{9}$ qui localise la fruiterie dans les offices. Outre que les fondations du château possèdent des murs épais d'un mètre au minimum, la fruiterie de Roissy est entourée sur ses quatre côtés de pièces qui contribuent à l'isoler du froid extérieur. La question de l'épaisseur des murs est donc réglée dans le cas d'étude présent.

L'exposition de la fruiterie est par sa position sans objet à Roissy.

La ventilation, sujet controversé, doit être envisagée. Seize auteurs recommandent une double porte d'entrée : que nous nous basions sur nos vestiges à Roissy, sur la lecture du plan du château de Champs-sur-Marne ou sur nos observations sur la fruiterie de la Reine dans les sous-sols du Grand Commun du château de Versailles, la mention de la double porte doit être comprise comme deux portes et non comme un sas tel celui conseillé pour accéder aux glacières. Les agronomes consultés recommandent un lieu sec, en tout cas non humide. Les auteurs préconisent bien de placer un «vaisseau » plat plein d'eau dans la fruiterie, mais uniquement pour évaluer le danger du gel. La petite cuve cylindrique 
observée à Roissy a-t-elle pu avoir cette fonction ? Ou bien au contraire, a-t-elle servi de réceptacle au « réchaud » rarement mentionné pour combattre le gel ? Une autre cuve à peu près similaire, accolée à une cheminée placée dans les offices du XVIII ${ }^{e}$ siècle, tendrait à confirmer la fonction de réceptacle pour une chaudière. Mais pourquoi l'avoir placée contre une paroi ? Ou bien faut-il voir dans cette cuve le piège à souris cité par quatre auteurs anciens?

Les fondations (F. 1573 et 1574) réalisées dans la continuité du mur porteur de la galerie des offices, positionnées au travers des deux accès à la fruiterie, formaient sans doute un obstacle très efficace à la pénétration des rongeurs.

Enfin, le critère de base pour identifier une fruiterie consiste en la présence de tablettes de bois, mentionnées par presque tous les auteurs ( 25 occurrences). Bien que les fouilles en milieu non humide livrent rarement des planches en bois, les scellements réalisés au sol ou dans les murs sont facilement repérables par les archéologues. Aménagements intérieurs ultimes et fragiles des fruiteries, leur fréquence confirme pourtant aisément l'interprétation. Ils ne manquent pas à Roissy.

Cinq auteurs ajoutent que des armoires sont parfaitement adaptées aux plus beaux fruits. Le placard aménagé dans la galerie a vraisemblablement eu cette fonction.

Si le devis de 1707 relatif à la construction du château ne détaille pas la fruiterie, toute maison de bon goût doit en ordonner une, au même titre que l'orangerie ou la glacière. Ces trois espaces offrent le luxe suprême de jouer avec le temps, de le forcer ou de le retenir ${ }^{10}$.

20 Le fruit est le dessert par excellence ${ }^{11}$. En Île-de-France, il existe une véritable arboriculture fruitière aristocratique. De nombreux nobles lisent les traités d'arboriculture et pratiquent eux-mêmes l'art de la taille. Les arbres fruitiers sont présents dans le projet de Le Nôtre pour les jardins de Roissy à la fin du XVII ${ }^{\mathrm{e}}$ siècle. Le comte de Caraman, grand amateur de jardins, embauche également un garde ${ }^{12}$ pour la surveillance des arbres fruitiers plantés le long des routes traversant la seigneurie de Roissy.

21 Il est donc logique de trouver une fruiterie dans le sous-sol du château de Roissy. Placée dans les offices, cette pièce de stockage est sous la direction de l'officier ${ }^{13}$.

Apprendre à distinguer archéologiquement une fruiterie d'une simple cave est un moyen d'affiner l'interprétation fonctionnelle et de mieux apprécier le statut des occupants d'un lieu.

\section{Les fruitiers de Montreuil-sous-Bois (Thalie Courelis)}

La ville de Montreuil-sous-Bois fut longtemps renommée pour son arboriculture fruitière et ses fameux « murs à la Montreuil ». Divers arbres fruitiers, en particulier les pêchers, sont cultivés en espalier le long de murs. En 1750, 600 des 800 ménages du village de Montreuil cultivent le pêcher ${ }^{14}$. La culture atteint une extension maximale durant la seconde moitié du XIXe siècle : la ville comportait alors $600 \mathrm{~km}$ de murs et $87 \%$ de sa population était constituée d'agriculteurs ${ }^{15}$. Grâce à son arboriculture spécialisée, Montreuil demeura la plus célèbre communauté de producteurs de fruits de luxe d'Europe du milieu du XVIII e au milieu du XXe s. Durant son apogée, la culture permit à la ville de produire jusqu'à 50000 pêches par hectare lors d'une bonne année, soit vingtdeux à vingt-cinq fruits par mètre courant de surface murale, et douze millions de fruits 
au total pour la récolte d'une année entière dans l'ensemble de la commune. Des quantités colossales de fruits arrivaient quotidiennement et en continu aux Halles de Paris durant la période de récolte des différentes variétés, qui s'étend au moins durant trois mois (de juillet à septembre) ${ }^{16}$. Les pêches de Montreuil furent également livrées à la Cour de France, d'Angleterre et de Russie. Afin de répondre à la demande constante du marché, les fruits étaient cueillis avant maturité et placés dans des fruitiers au sein des maisons des horticulteurs. Ces fruitiers abritaient au long de leurs murs des étagères en bois sur lesquelles étaient entreposés les fruits avant leur vente.

\section{Les fruitiers observés à Montreuil}

Une étude sur les maisons des horticulteurs montreuillois ${ }^{17}$ fut menée durant l'année universitaire 2008-2009. Parmi les maisons d'horticulteurs étudiées, six ${ }^{18}$ nous apportent des informations pratiques sur les fruitiers (fig. $\mathbf{n}^{\circ} \mathbf{4}$ ).

Figure 4

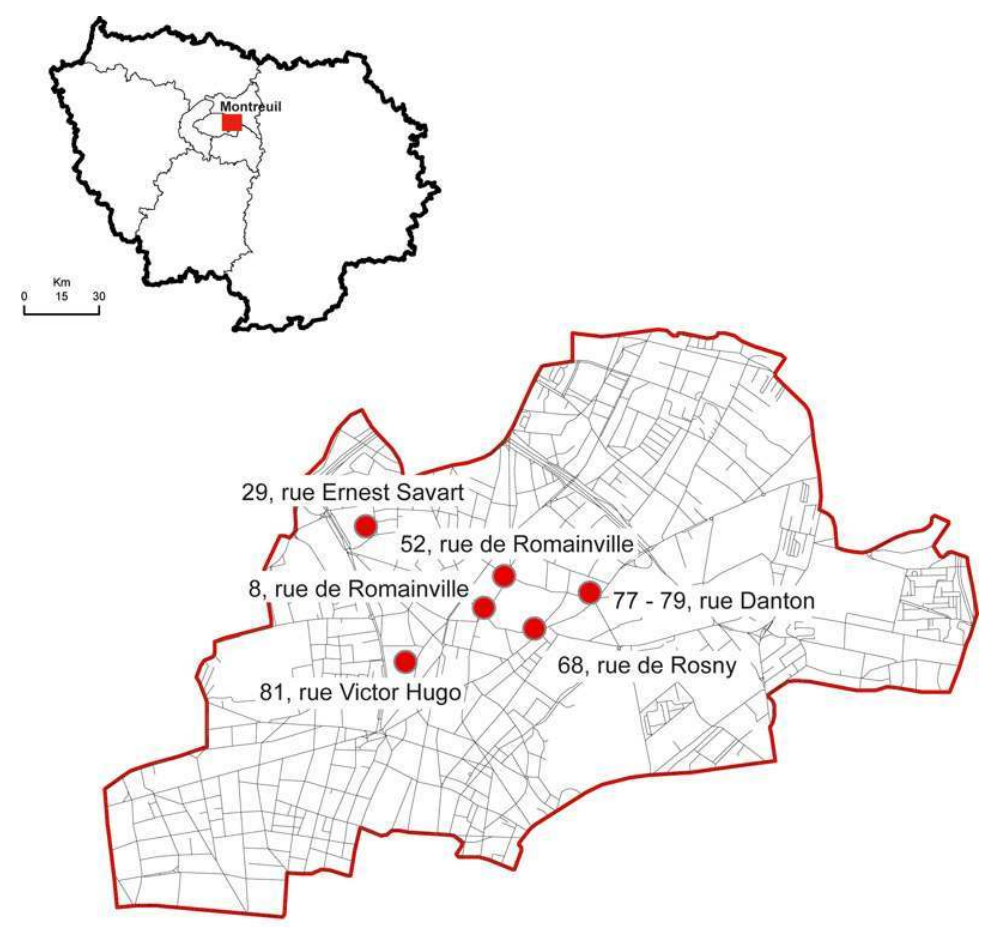

Fig. 4 : localisation des maisons d'horticulteurs étudiées à Montreuil-sous-Bois.

Localisation des maisons d'horticulteurs étudiées à Montreuil-sous-Bois.

Phot. Courelis, Thalie, Belarbi, Mehdi. (c) Inrap 2010.

La maison située au 68 rue de Rosny possédait un fruitier au grenier, accessible par une porte (orientée vers le nord-est) depuis la maison, et ouvert sur la rue par une fenêtre (nord-ouest). Cette dernière est encore aujourd'hui surmontée d'une poulie (fig. $\mathbf{n}^{\circ} 5$ ). La date de construction de cette maison n'est pas connue avec précision, cependant elle apparaît sur les cartes anciennes entre 1740 et 1837. 
Figure 5

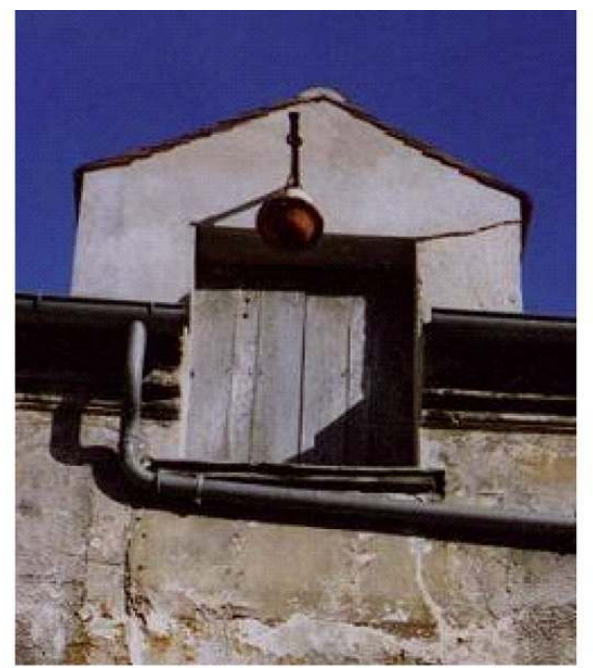

Fig. 5 : 68 rue de Rosny, détail de l'ouverture du grenier, au-dessus de laquelle subsistent les traces d'une poulie

68 , rue de Rosny, détail de l'ouverture du grenier, au-dessus de laquelle subsistent les traces d'une poulie.

Phot. Courelis, Thalie, 2010. (c) Courelis, Thalie.

Les maisons situées aux 77 et 79 rue Danton datent de 1860. Elles ont un plan symétrique similaire. Sur chaque parcelle deux bâtiments parallèles entre eux et perpendiculaires à la rue, se font face. L'un abrite les locaux d'habitation, l'autre divers locaux tels que fruitier, écurie, clapier/poulailler, etc. Deux fruitiers sont positionnés avec certitude dans la maison du 79 : l'un au premier étage de l'un des bâtiments annexes, l'autre au grenier de l'habitation. Le fruitier au grenier nous est connu par le témoignage de sa propriétaire. Sa porte d'accès est exposée au nord. Une poulie et une corde servaient à monter au

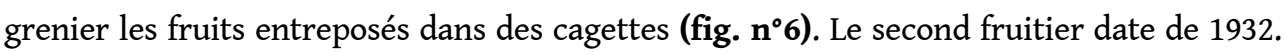
Disposée à l'étage d'une annexe, pénétrable par une porte large de $72 \mathrm{~cm}$ exposée au nord, la pièce mesure 5,1 x 3,15 m. Deux fenêtres ouvertes côtés sud et ouest participent à la ventilation. L'un des murs est mitoyen, deux autres murs extérieurs sont épais de $20 \mathrm{~cm}$. 
Figure 6

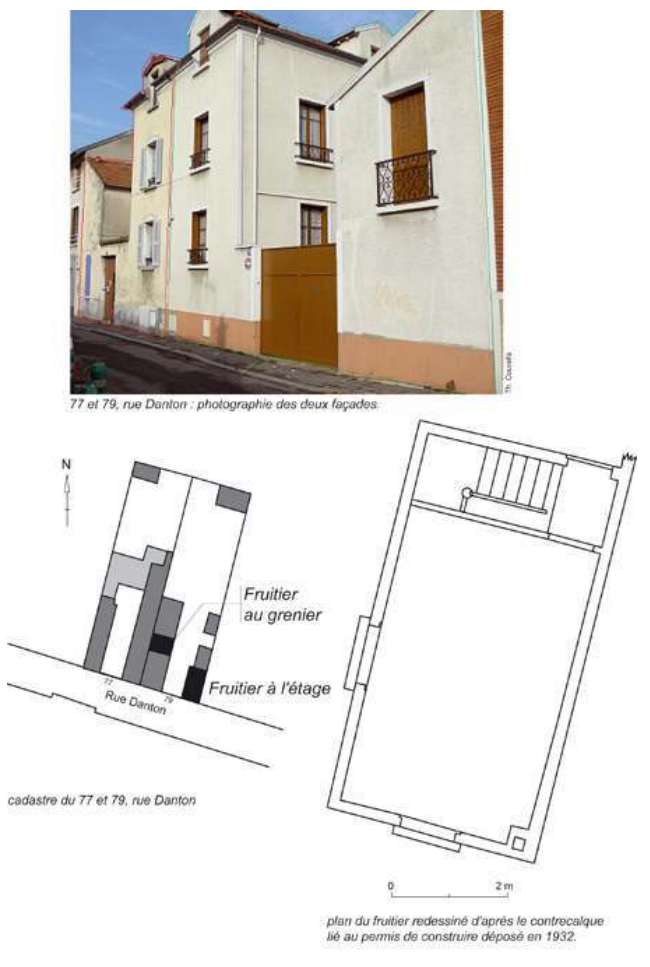

Fig. 6 : plan et localisation de la maison du 79, rue Danton à Montreuli-sous-Bois.

Plan et localisation de la maison du 79, rue Danton à Montreuil-sous-Bois.

Phot. Courelis, Thalie, Belarbi, Mehdi. (C) Inrap 2010

La maison située au 8 rue de Romainville (fig. $\mathbf{n}^{\circ} 7$ ), construite en $1903^{19}$, abrite en soussol un fruitier bien conservé avec toutes ses étagères en bois. La pièce mesure 4 x 8,5 m. Elle est accessible depuis la maison par une porte ouvrant vers le nord-est, et depuis l'extérieur par un soupirail donnant sur la rue (vers le nord-ouest). Une fenêtre donne sur le jardin côté sud-est. 


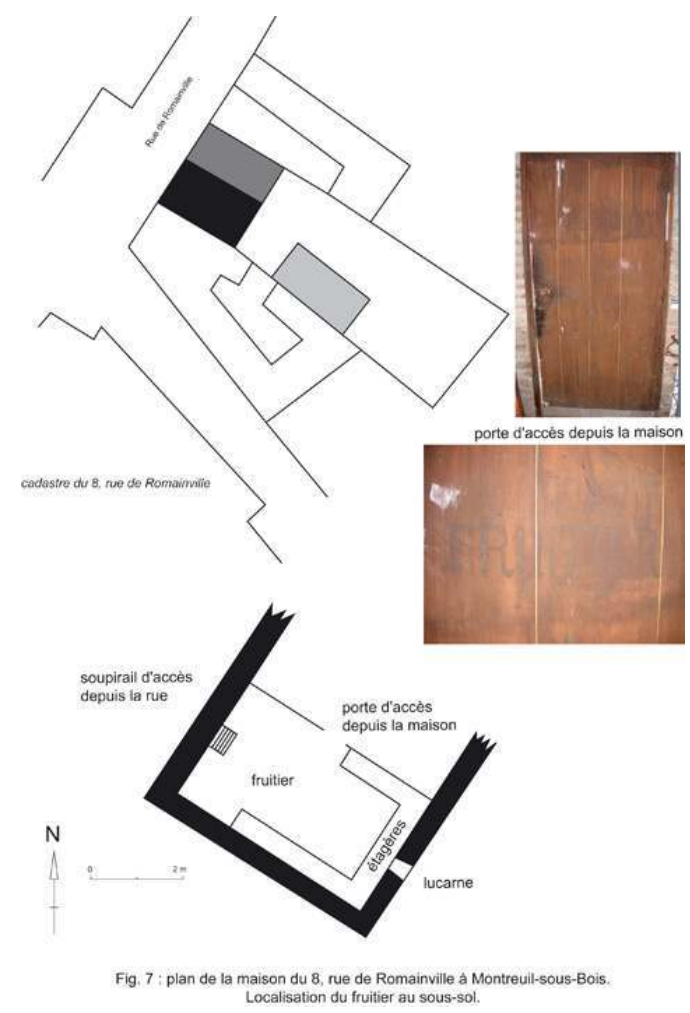

Plan de la maison du 8, rue de Romainville à Montreuil-sous-Bois. Localisation du fruitier au sous-sol. Phot. Courelis, Thalie, Belarbi, Mehdi. (c) Inrap 2010.

La maison de culture du 29 rue Savart date des années 1920. Observée par l'Écomusée de La Courneuve avant sa démolition en 2001, cette propriété a fait l'objet d'une étude ethnographique poussée. Elle comprenait deux fruitiers, l'un était disposé dans la cave et une sous une partie du pavillon, l'autre occupait tout le rez-de-chaussée d'un bâtiment annexe ouvert au sud (vers la maison). Un grenier participait à l'isolation de ce fruitier.

La maison située au 52 rue de Romainville (fig. n8) est datée de 1929. Sur le plan déposé lors du permis de construire ${ }^{20}$, le fruitier localisé au sous-sol mesure 3,7 x 3,25 m. Une porte d'accès (sud-est) depuis la maison et une petite fenêtre sur rue (nord-ouest) mesurent chacune un mètre de large. Le mur côté nord mesure $40 \mathrm{~cm}$ d'épaisseur, les autres $25 \mathrm{~cm}$. 


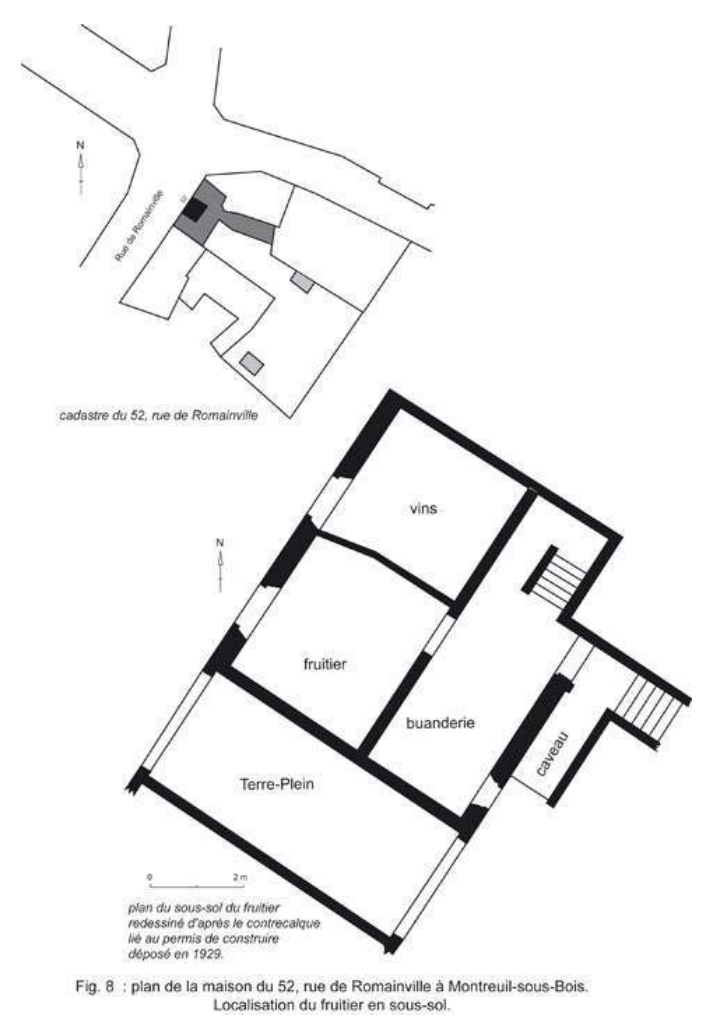

Plan de la maison du 52, rue de Romainville à Montreuil-sous-Bois. Localisation du fruitier en sous-sol. Cartog. Courelis, Thalie, Belarbi, Mehdi. (c) Inrap 2010.

La maison située au 61 rue Victor Hugo a possédé au moins un fruitier au grenier. La pièce mesure environ $6 \times 5 \mathrm{~m}$, est accessible par une porte (nord) et était percée par une fenêtre, aujourd'hui supprimée, donnant sur l'arrière de la maison (ouest).

\section{Confrontation entre les fruitiers observés et la théorie agronomique sur leur construction}

31 La pratique de la maison horticole de Montreuil répond-elle à la théorie de la construction agricole du fruitier, telle que donnée depuis le XVIII ${ }^{e}$ siècle par les agronomes?

Plusieurs critères sont mentionnés par ces auteurs au sujet des fruitiers : emplacement de la pièce dans la maison, son atmosphère (plutôt sèche ou humide, froide ou chaude, etc.), exposition, nombre d'ouvertures, épaisseur des murs et encore aménagement intérieur.

Jusque dans les années 1785 , les auteurs situent de préférence le fruitier au rez-dechaussée, plus rarement au premier étage, mais jamais au sous-sol ni au grenier. Puis à partir de cette date, le meilleur emplacement est déplacé au sous-sol, sauf pour Forney qui, en 1863, préconise le rez-de-chaussée ou le premier étage. Notons que cet auteur nous informe de la pratique montreuilloise. À leur manière, l'abbé Rozier $(1784, \mathrm{~V})$ et Paquet (1844) rappellent que l'emplacement du fruitier reste un sujet controversé.

Parmi toutes ces maisons étudiées, maisons de culture pourtant regroupées sur le même terroir et parfois la même période chronologique, on note que le fruitier peut trouver 
place dans toutes les parties de la maison (3 fruitiers en sous-sol, 1 au rez-de-chaussée, 1 à l'étage et 3 au grenier), preuve qu'au début du XX $\mathrm{XX}^{\mathrm{e}}$ siècle encore, il n'y a pas de règle fixe sur son emplacement. Celui-ci n'est donc pas déterminant ou si important, malgré la place accordée par les auteurs à débattre du sujet.

Aucun réel élément de comparaison concernant l'atmosphère dans les fruitiers des maisons visitées ne peut être apporté, néanmoins l'actuelle propriétaire de la maison au 79 rue Danton mentionne un poêle disposé dans le fruitier en cas de température trop basse. Seul Mozard évoque ce procédé en 1814, Bastien (1798) et Liger (1777) mentionnant bien qu'on ne fasse pas de feu dans la fruiterie.

Les dimensions, l'orientation et le nombre des ouvertures sont documentés sur les maisons de Montreuil. L'exposition des fruitiers est un sujet délicat, abondamment traité jusqu'au début du XIX siècle, avant de disparaître. Largement intégrés dans des maisons, les fruitiers de Montreuil ont toutefois un ou deux côtés en prise avec l'extérieur. Cette exposition « relative » des fruitiers privilégie l'Est. On observe toutefois également des pans de murs au Nord, à l'Ouest ou au Sud. Le seul fruitier entièrement isolé d'une habitation a été observé rue Savart: il se présente sous la forme d'un rectangle axé estouest, donc exposé au sud et au nord. L'exposition au sud, ici renforcée par la présence de deux portes, est celle préférée par les auteurs du XVIII siècle, déconseillée par Paquet, le dernier auteur qui en 1844 aborde le sujet.

Les ouvertures observées à Montreuil répondent-elles aux exigences des agronomes?

Aucune double porte n'est présente, malgré leur récurrence ( 15 mentions) parmi les auteurs anciens. Les fenêtres suffisaient sans doute à la ventilation. Notons toutefois qu'un fruitier ne possédait aucune fenêtre. Quatre des sept portes observées s'ouvrent côté nord, trois côté est ; les expositions les plus fraîches seraient-elles recherchées ? Les fenêtres en relation directe avec l'extérieur privilégient des expositions à l'ouest (5 mentions), preuve qu'on ne craint plus l'humidité. Trois cas d'ouverture au nord et trois au sud tendent à confirmer l'absence d'homogénéité des fruitiers de Montreuil et l'absence de risques réels pour la préservation des fruits.

Une bonne épaisseur de murs, préservant des variations de températures extérieures, est l'un des autres critères constamment évoqués par les agronomes pour conserver au mieux les fruits. Les auteurs conseillent l'emploi de murs épais de deux à quatre pieds ( 65 à $130 \mathrm{~cm}$ ). Là encore, la réalité observée ne correspond pas aux conseils donnés par les agronomes, car aucun mur relevé ne mesure plus de $40 \mathrm{~cm}$ d'épaisseur. La plupart des murs ont une épaisseur entre $8 \mathrm{~cm}$ (simple cloison) et $25 \mathrm{~cm}$. On est donc bien loin des « murs épais » préconisés.

40 En guise de conclusion sur les maisons de Montreuil, il semble que l'emplacement du fruitier dans la maison importe peu, pourvu qu'il ne soit ni trop chaud, ni trop froid, ni trop sec, ni trop humide, ni trop ventilé. Il s'agit simplement de trouver un compromis entre toutes ces possibilités : si la cave est trop humide, il sera placé à un autre niveau, de même si le grenier est trop sec ou trop chaud, etc.

41 Comment expliquer les différences observées entre les conseils donnés par les auteurs et la réalité observée dans les maisons visitées? Les cultivateurs ont peut-être déduit euxmêmes un certain nombre de critères suite à leur expérience personnelle, et ont tout simplement placé le fruitier dans la maison " là où il y avait de la place » à proprement parler, c'est-à-dire à l'emplacement le plus fonctionnel par rapport à l'agencement global de la maison. 
Une comparaison avec un nombre plus important de fruitiers apporterait des informations supplémentaires.

\section{Les lieux du stockage des fruits à pépins dans les maisons paysannes d'île-de-France (circa 1650-1830) décrits au moyen de quelques caractéristiques variables (Jean-Jacques Péru)}

Les sources les plus accessibles, celles qui permettent de connaître d'une façon sérielle, quasi statistique, la façon dont les paysans d'Île-de-France utilisaient leur maison à des fins de conservation fruitière sont classiques. Ce sont les actes de la pratique, tout spécialement les inventaires dressés lors de la dissolution des communautés lesquels prisent de manière détaillée les stocks alimentaires ou commerciaux détenus par les ménages au moment du décès de l'un de ses membres.

296 inventaires échelonnés entre 1650 et 1830 de stockeurs de fruits frais (pommes et poires destinées à la consommation familiale ou à la vente au marché) ont été extraits d'une base composée de plusieurs milliers d'inventaires après décès recueillis dans une zone comprise entre Chartes et Beauvais, Provins et La-Roche-Guyon. Comment conservait-on ces fruits destinés à être consommés non-cuits ou séchés? Comment contrôlait-on leur maturation? Jusqu'à quel point savait-on éviter leur trop rapide pourrissement?

Sept fois sur dix, on a uniquement affaire à des stockeurs de pommes «à couteau ", le dernier petit tiers de notre échantillon se partageant également entre détenteurs de pommes et poires conjointement, de poires uniquement et de quelques vignerons également « cidriculteurs ", producteurs de cidre "pommé » ou " poiré » selon les cas. Ces pommes et poires destinées à être broyées seront simplement mentionnées dans cette note. Une mise en garde préliminaire : l'espèce et la variété des fruits conservés jouaient un rôle déterminant dans les stratégies de conservation mises en œuvre par les paysans, mais on toucherait ici à une autre question, disons simplement que les performances globales de conservation de nos fruits à pépins sont très honorables, puisque des fruits encore dotés de valeur commerciale ou encore consommables à l'état frais sont prisés jusque dans la seconde quinzaine de mai et ce quelle que soit l'époque considérée, du XVII ${ }^{e}$ siècle au premier tiers du XIX ${ }^{e}$ siècle.

Il s'agira de s'interroger sur les stratégies paysannes de conservation des fruits climactériques par le truchement de leurs maisons appréhendées ici comme de véritables "machines passives $»^{21}$ dotées non pas tant de fonctionnalités que de «fonctionnements » avec leurs pièces à feu, à froid, à sec, à humidité, à confinement, à ventilation...

Nous dénommons fruits climactériques, des fruits qui lorsqu'ils sont cueillis au bon moment - Louis Aubin, arboriculteur réputé de Montreuil-sous-Bois disait de ces pommes et poires qu'elles devaient être récoltées bien "octobrées » - et placés dans de bonnes conditions peuvent se conserver jusqu'en mai, ce qui est le cas en Île-de-France.

Caractéristique I. Le calendrier du stockage ${ }^{22}$ des fruits climactériques (pommes et poires) chez les paysans d'Île de France : esquisse d'un modèle saisonnier 


\begin{tabular}{|l|l|l|l|l|}
\hline & $1790-1830, \mathrm{~N}=89$ & $1730-1788, \mathrm{~N}=106$ & $1600-1728, \mathrm{~N}=101$ & Ensemble \\
\hline fin août et septembre & $6 \%$ & $3 \%$ & $7 \%$ & $5 \%$ \\
\hline otobre & $11 \%$ & $12 \%$ & $16 \%$ & $13 \%$ \\
\hline novembre et décembre & $52 \%$ & $40 \%$ & $43,5 \%$ & $45 \%$ \\
\hline janvier-février & $27 \%$ & $34 \%$ & $25,5 \%$ & $29 \%$ \\
\hline mars à mai & $4 \%$ & $11 \%$ & $8 \%$ & $8 \%$ \\
\hline
\end{tabular}

\begin{tabular}{|l|l|l|l|l|l|l|}
\hline vignerons & cultivateurs & $\begin{array}{l}\text { laboureurs } \\
\text { et fermiers }\end{array}$ & $\begin{array}{l}\text { marchands } \\
\text { (dont } \\
\text { «fruitiers ») }\end{array}$ & jardiniers & manouvriersjournaliers & inconnu \\
\hline 153 & 51 & 32 & 18 & 18 & 9 & 15 \\
\hline
\end{tabular}

Pour les siècles considérés, les stockeurs de fruits climactériques sont essentiellement des vignerons, des petits «cultivateurs » au début du XIX ${ }^{e}$ siècle selon la terminologie mise en place sous la Révolution et des «manouvriers » ou journaliers $(213 / 296=72 \%)$. C'est donc de la maison vigneronne ou plus largement paysanne, voire villageoise, comme lieu de conservation fruitière qu'il faut nous occuper afin d'y porter au jour les « machineries » de stockage.

Dans une zone géographique où il n'est peut-être pas de petit cultivateur qui ne soit d'abord vigneron et inversement de vigneron qui ne soit avant tout un petit "polyculteur", le poids écrasant de la maison vigneronne ne doit cependant pas estomper le rôle des fermes, des maisons villageoises où habitaient les «marchands fruitiers » et des châteaux ou maisons de campagne bourgeoises dont les vergers étaient entretenus par des jardiniers partiellement rétribués au moyen d'un intéressement au produit des récoltes. En fait le stockage des fruits climactériques frais est un problème universel à tous les habitats ruraux d'île de France, mais avec une écrasante majorité un problème paysan.

Où y conserve-t-on les pommes et poires?

53 Au point de dégrossissage atteint maintenant il devient indispensable de tenter d'ouvrir la «boîte noire » des «fruitiers ", ce qui en l'état de la documentation dépouillée ne peut se faire que par le truchement d'une approche monographique des "jardiniersvignerons » de Montreuil-sous-Bois. En effet, d'après nos sources - ou plutôt notre échantillon - le mot même de «fruitier » entendu comme lieu spécial, n'apparaît qu'au milieu du XVIII ${ }^{\mathrm{e}}$ siècle : en 1742, chez Jean Mourette, jardinier au Château de Juilly ${ }^{23}$, puis en 1748 chez Jean Mercier ${ }^{24}$, laboureur à la Villette aux Aulnes à Mitry et concomitamment - chez Jean Baptiste Picot, vigneron à Montreuil-sous-Bois en décembre de la même année ${ }^{25}$. On tient là nos trois sous-populations de stockeurs en "fruitier " 
telles que révélées par notre échantillon (il manque hélas l'observation de Thomery) : les jardiniers de château, les fermiers et les vignerons-jardiniers de Montreuil qui détiennent 19 des 24 fruitiers ( 8 sur 10) de notre population de stockeurs, alors qu'ils ne pèsent que $14 \%$ de celle-ci. D'après nos sources, les «marchands fruitiers » du XVII ${ }^{e}$ siècle stockant selon les techniques du temps ignorent le «fruitier ». Pour l'instant, je ne définis pas la notion. Statistiquement, le « fruitier » comme lieu particulier n'a que peu de poids.

Malgré l'imprécision des sources, 4 fois sur 10, le lieu exact du stockage fruitier n'est pas indiqué - il se dégage de l'analyse du reste de notre échantillon une impression assez nette. Il existe une sorte de topographie fruitière des maisons rurales où deux profils de stockeurs se distinguent : ceux qui stockent tout en bas et rien à l'étage (ils sont 101) et ceux qui conservent en haut (ils sont 66) en utilisant plus rarement - moins d'un sur dix) le bas de leurs maisons.

Dans un article pionnier, Marcel Lachiver avait dressé la carte des maisons vigneronnes de l'ex-Seine et Oise, selon qu'elles possédaient ou non des étages supérieurs. Il est d'évidence que pour stocker en étage il faut déjà en posséder un! Mais nos 20 stockeurs en grenier ne livrent qu'un seul stockage concomitant en cellier - là où cuves et futailles et bientôt (après 1760) pommes de terre sont réservés. Il s'agissait donc d'un choix. Ainsi, je remarque par exemple que ces 20 utilisateurs du grenier sont très peu cidricoles $(2 / 20)$. Les fruits y étaient-ils étendus "épars " plutôt qu'en tas? L'examen des modes et techniques de conservation fruitière selon les niveaux de la maison renforce notre impression d'orientations spécifiques dans le choix des localisations de stockage. Il y avait à l'évidence une appropriation des lieux selon les effets recherchés.

En terme de contrôle de la maturation, de visite périodique des lieux, de soustraction des fruits pourris, de protection, confinement, aération, bref de contrôle de la respiration de nos fruits climactériques, on n'en saura guère plus au moyen de nos seules sources. C'est d'ailleurs ici que commence l'enquête ethnographique. Une étude plus complète que cette simple esquisse, réunissant d'un même élan ethnographie de terrain et archéologie du bâti, se pencherait tout à la fois sur les systèmes de liaison haut/bas équipant nos maisons paysannes : escalier en - ou hors œuvre, échelles intérieures ou non, trappes et trémies, cordes et poulies, etc. comme sur la nature des matériaux des planchers: plâtre plutôt que parquets et se soucierait des couvertures et sous-couvertures isolantes, etc. ${ }^{26}$

Resserrons maintenant l'analyse autour de cinq zones de stockage ou de production fruitière spécialisée variablement cidricole. Soit les jardiniers-vignerons de Montreuilsous-Bois, les vignerons du Hurepoix, ceux de la vallée de Montmorency, ceux de Brie et ceux de sud-Picardie. Cinq grands groupes de caractéristiques ont été déterminés selon que les fruits étaient stockés en BAS ou en HAUT de la maison, selon les regroupements d'ESPECE, selon le MODE de stockage et l'UNITÉ de compte utilisée lors de la prisée. 


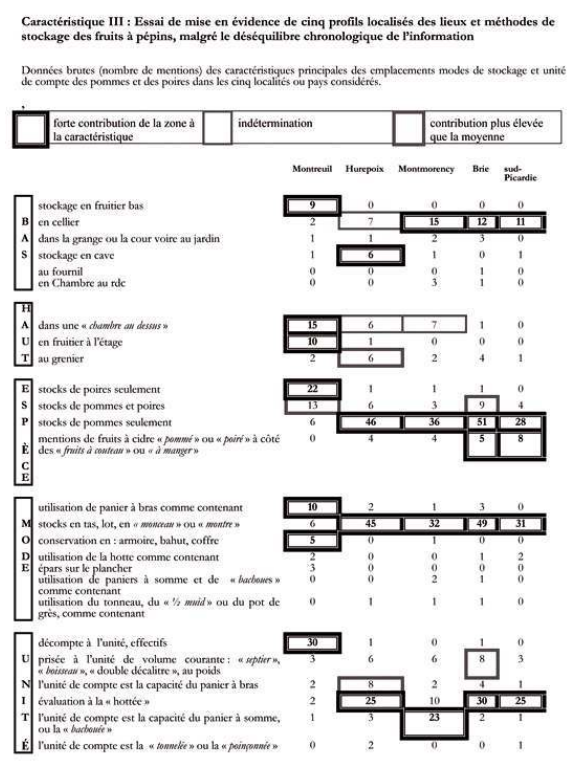

Sauf pour la vallée de Montmorency où une nette propension statistique à l'utilisation de la «bachouée » comme unité de compte peut être notée, c'est le profil Montreuillois qui détermine tous les autres. Si l'on rencontre intra-régionalement des pays fruitiers, des « vallées » ou des « montagnes » comme le pays de Goële par exemple (non étudié encore à ce jour), Montreuil est, à partir des années 1730, un village fruitier spécialisé tout comme Thomery qui n'est pas étudié dans cet article. C'est de plus un très gros bourg et un vaste territoire. Il est donc logique d'y rencontrer une concentration de signes particuliers, moins diffus que certains traits communs.

Rappelons l'absence de l'appellation spécifique du fruitier en tant que tel avant le milieu du XVIII siècle à Montreuil, mais un emploi de la chambre haute ici beaucoup plus qu'ailleurs. Reste à comparer ce que les sources permettent de saisir de l'aménagement voire de l'équipement fruitiers de ces deux emplacements. Ce qui définira le "fruitier » jusqu'à présent simple lieu désigné de façon spéciale.

$\mathrm{Au}$ XVII siècle les vignerons de Montreuil stockent leurs pommes en tas, au rez-dechaussée ou en bas dans leurs celliers comme la plupart de leurs homologues régionaux. Le stockage rejoint l'étage supérieur au XVIII ${ }^{e}$ siècle et au début du XIX ${ }^{e}$ siècle.

On a globalement 57 stockeurs en chambre à fruit située à l'étage et en «fruitier » haut (en effet: dans 8 cas sur 10 le «fruitier» ou la chambre à fruit sont à l'étage). Nos deux sous-populations sont à peu près d'effectif égal.

Si nous comparons l'équipement mobilier des chambres hautes aux "fruitiers" en général, pour la période considérée, c'est la «tablette " arrangée en "pourtour » qui caractérise le «fruitier». C'est ce qui permet le plus commodément le traitement individuel des fruits. Chez les paysans ce mode de conservation est tardif et étroitement localisé. Mais on ne peut dire s'il s'agit du résultat d'une influence littéraire (toujours possible) ou du remploi du matériel de séchage des fromages, la première spécialisation des montreuillois. En effet, l'emblématique panier à fruits de Montreuil, le «noguet » ou 
"panier plat», un contenant de stockage fruitier en chambre haute non-équipée de " tablettes ", était au XVII ${ }^{\text {e }}$ siècle, tout bonnement un " panier à fromage » comme nous l'apprend l'inventaire de Martine Boudin, femme de Pierre Vitry, dressé le 8 août $1665^{27}$. Or partout en île-de-France, les fromages étaient stockés sur des planches. Quand à l'influence littéraire, si en $1759^{28}$, Jean Patron, vigneron à Crégy (Seine-et-Marne), possédait un exemplaire des « instructions pour les arbres fruitiers », de La Quintinie, son cellier était garni d'un très ordinaire « tas de pommes contenant 6 hottées de differents prix... » selon l'usage éprouvé du pays.

Caractéristique IV : Des aménagements et modes de stockages fruitiers en chambre chez les Montreuil et chez les autres. Essai de modèle fonctionnel

\begin{tabular}{|c|c|c|c|}
\hline aménagements, mode et unité de stockage. & à Montreuil & chez les autres & lieu \\
\hline stocks en tas, lot, en «monceau » ou « montre» & 0 & 22 & c \\
\hline évaluation à la « hottée » & 0 & 6 & H \\
\hline évaluation à la « somme à cheval » & 1 & 2 & A \\
\hline mention de planches & 0 & 1 & M \\
\hline décompte à l'unité, effectifs & 0 & 1 & B \\
\hline dans un coffre & 0 & 0 & $\mathbf{R}$ \\
\hline contenus dans une «manette» & 1 & 0 & E \\
\hline dans un « cueilloir» & 1 & 0 & \\
\hline dans un «noguet» & 2 & 0 & $\mathrm{H}$ \\
\hline «épars» sur le plancher & 3 & 0 & A \\
\hline dans un panier à bras & 4 & 1 & $\mathrm{u}$ \\
\hline & & & $\mathbf{T}$ \\
\hline \multirow{3}{*}{ DANS UNE ARMOIRE } & 3 & 1 & E \\
\hline & & & \\
\hline & 1 & 0 & \\
\hline « tablette, tablettes à fruit» & 8 & 1 & \\
\hline \multirow[t]{2}{*}{ mention de «tréteaux» et « montant» } & 7 & 1 & $\mathbf{F}$ \\
\hline & & & $\mathbf{R}$ \\
\hline «marchepied» & 4 & 0 & $\mathbf{U}$ \\
\hline
\end{tabular}




\begin{tabular}{|l|l|l|l|}
\hline «pourtour » & 2 & 0 & I \\
\hline «planches de voliges " & 3 & 0 & T \\
\hline «planches " & 3 & 2 & I \\
\hline «planches en tablettes » & 2 & 0 & E \\
\hline « rayons de planches " & 2 & 0 & R \\
\hline "grandes planches en sapin » & 1 & 0 & \\
\hline total des mentions de « planches " & $\mathbf{1 2}$ & 2 & \\
\hline
\end{tabular}

Cette approche sérielle et modélisatrice d'un usage particulier - plutôt que fonction : les bâtiments ruraux remplissaient une multitude de «fonctions " sans que les «cahiers des charges " nous en soient suffisamment connus - des maisons paysannes d'île-de-France aura eu en premier lieu l'utilité de fournir un cadre à l'interprétation ethnographique et archéologique des pratiques de conservation fruitière de plus ou moins longue durée historique observées à Montreuil-sous-Bois qui sont dès lors pourvues d'un large contexte chronologique et régional. Cette approche délibérément statistique (et donc aride) aura eu aussi le mérite d'aider à l'exploitation de la littérature agronomique ancienne souvent contradictoire dans ses descriptions/prescriptions. Enfin, cette démarche de décomposition élémentaire, puis de recomposition dynamique des différents éléments caractérisés au cours de cette étude, bref cet essai de modélisation, aura, nous l'espérons, montré toute la complexité et la cohérence de pratiques paysannes révolues, mais inscrites dans la pierre si l'on peut dire, dès lors qu'on voudra bien leur consacrer tout le temps de recherche et d'analyse nécessaires.

\section{Conclusion générale}

Par un effet de "miroir» social, la littérature agronomique ancienne, ignorant les méthodes paysannes de conservation des fruits à pépins (en « tas ", principalement mais pas seulement) nous renseigne presque exclusivement sur un mode de stockage en espace confiné où les fruits posés sur des tablettes sont traités individuellement. Cette pièce particulière est dénommée fruitier. Et, comme attendu - entre 1650 et 1830 - les archives manuscrites ne nous signalent de fruitier que dans les châteaux, les grosses fermes ou les belles demeures bourgeoises. À une exception près cependant, le village de Montreuilsous-Bois où, après-1750, se généralise une méthode de conservation des pommes et des poires de " choix $»^{29}$ qui, non sans rappeler certaines des prescriptions des manuels, s'en éloigne cependant assez pour qu'on puisse parler de «fruitiers à la Montreuil». Ces fruitiers vernaculaires ont été observés lors d'une campagne ethnographique de "sauvetage ", que corrobore bien l'enquête historique. Mais ces deux approches doivent être complétées par une analyse archéologique du bâti afin de mieux comprendre ces derniers bâtiments témoins d'une arboriculture fruitière paysanne originale. 


\section{BIBLIOGRAPHIE}

Sources imprimées utilisées pour le tableau 1.

AUBERT DE LA CHESNAYE DES BOIS, François-Alexandre. Dictionnaire universel d'agriculture et de jardinage, de fauconnerie, chasse, pêche, cuisine et manège. Á Paris : chez David, 1751, 2 volumes, 730 p. et 467 p.

BASTIEN, Jean-François. La nouvelle maison rustique ou Économie rurale pratique et générale de tous les biens de Campagne. Tome II, 1798, 899 p.

BONA, Théodore. Manuel des constructions rurales. Paris : Librairie agricole de la maison rustique, $1873,296 \mathrm{p}$.

BRETONNERIE (M. de La). L'école du jardin fruitier. Paris, 1784, 2 tomes, 546 et 658 p.

BRISSEAU de MIRBEL, Charles-François. « De la conservation des fruits ». Dans BAILLY, BIXIO et MALPEYRE (dir.). Maison rustique du XIX ${ }^{e}$ siècle, tome 1, 1834, p. 330.

DAVILER, Auguste-Charles. Dictionnaire d'architecture civile et hydraulique. Paris : Chez Ch. A. Jombert, 1755, $366 \mathrm{p}$.

DE BONNEFONS, Nicolas. Le jardinier françois. Á Amsterdam : Chez Raphaël Smith, 1661, 379 p.

DE LA RIVIERE \& DU MOULIN. Méthode pour bien cultiver les arbres à fruit et pour élever les treilles. Á Utrecht : Chez Étienne Neaulme, 1739, 232 p.

DE NEUFCHATEAU, François. Dictionnaire d'agriculture pratique. Paris : Aucher-Éloi et V. de Boisjoslin Libraires-éditeurs, 1828, 2 tomes, 594 p. et 779 p.

DIDEROT, Denis \& D'ALEMBERT, Jean le Rond. Encyclopédie ou dictionnaire raisonné des sciences, des arts et des métiers. Troisième édition, Genève : Jean-Léonard Pellet, 1782. T. XV, 984 p.

DEMARSON, Madame. Guide de la ménagère ; Manuel complet de la maîtresse de maison. Bruxelles, 1838. Tome I, $268 \mathrm{p}$.

DU BREUIL, Alphonse. Cours élémentaire théorique et pratique d'agriculture. Paris : Victor Masson, 1846, 613 p.

FORNEY, Eugène. Le jardinier fruitier. Paris : chez l'auteur, place royale, 1863, 308 p.

LA QUINTYNIE, Jean-Baptiste. Instructions pour les jardins fruitiers et potagers. À Paris : chez Despilly, 1739. Tome II, $587 \mathrm{p}$.

LE CAMUS DE MEZIERE, Nicolas. Le génie de l'architecture ou l'analogie de cet art avec nos sensations. À Paris : chez Benoit Morin imprimeur-libraire, 1780, 276 p.

LELIEUR DE VILLE-SUR-ARCE, Comte de. La pomone française ou traité des arbres fruitiers taillés et cultivés d'après la fructification et la végétation particulière à chaque espèce. Paris : $\mathrm{H}$. Cousin libraireéditeur, 1842, 541 p., $18 \mathrm{pl}$.

LIGER, Louis. La nouvelle maison rustique. À Paris : chez Desaint, 1777. Tome II, 756 p.

MARTIN, Jean-Baptiste et ROY, Julien. Agriculture et jardinage. Principes scientifiques et applications. Paris : Delalain frères, 1900, 280 p. 
MILLET-ROBINET, Cora. Maison rustique des dames. $14^{\mathrm{e}}$ édition. Paris : Librairie agricole de la maison rustique, 1893,681 et $664 \mathrm{p}$.

MOZARD, Jean. Principes pratiques sur la culture des arbres fruitiers et principalement du pêcher. Paris, $1814,160 \mathrm{p}$.

PAQUET, Victor. Traité de la conservation des fruits. Paris : H. Cousin éditeur, 1844, 308 p.

PARMENTIER, Antoine-Augustin. Économie rurale et domestique. Paris : Bibliothèque universelle des dames, 1788. Vol. II, $248 \mathrm{p}$.

PERTHUIS de LAILLEVAULT, Léon. Traité d'architecture rurale. Paris, 1810, 263 p., 25 pl.

PLUCHE, Noël-Antoine. Le spectacle de la nature. Paris : chez la Veuve Étienne, 1741. Tome II, 468 p. ROZIER, François (Abbé). Cours complet d'agriculture. Á Paris, rue et hôtel Serpente, 1784. Tome V, $747 \mathrm{p}$.

RUENEUVE, Angran de. Observations sur l'agriculture et le jardinage. Paris, 1712. 2 tomes, 384 et $406 \mathrm{p}$.

SAINT-FÉLIX-MAUREMONT, Armand-Joseph (Marquis de). Architecture rurale, théorie et pratique, à l'usage des propriétaires et des ouvriers de la campagne. $2^{\mathrm{e}}$ édition. Toulouse, 1826, 395 p., pl.

VARRON, Marcus Terentius. De l'agriculture. Traduction de M. Wolf publié par Firmin Didot en 1864 sous la direction de M. Nisard. Paris : Éditions Errance, 96 p.

\section{NOTES}

1. - Nous n'aborderons pas ici les autres techniques de conservation des fruits dans des contenants remplis de pulvérulents (sciure, balle...), conservation par enduit, séchage, immersion ou blanchiment.

2. - QUELLIER, Florent. Des fruits et des hommes. L'arboriculture fruitière en Ile-de-France (vers 1600vers 1800). Rennes : Presses Universitaires de Rennes, 2003, p. 89.

3. - PAQUET, Victor. Traité de la conservation des fruits. Paris : H. Cousin éditeur, 1844, p. 122 et suivantes.

4. - GAUTIER, Michel. «La conservation en fruitier ». Jardins de France, sept. 2001, $\mathrm{n}^{\circ} 7$, p. 38-41.

5. - Signalons que cette cave est réduite de moitié par rapport au siècle précédent.

6. - F. 1574.

7. - F. 1136.

8. - F. 1669.

9. - Le château de Champs-sur-Marne est chronologiquement, architecturalement et sociologiquement très proche de celui de Roissy.

10. - QUELLIER, Florent. Des fruits et des hommes. L'arboriculture fruitière en Ile-de-France (vers 1600vers 1800). Rennes : Presses Universitaires de Rennes, 2003, p. 222.

11. - Il se consomme en compote, en dragées, massepain, confiture, eau-de-vie... (QUELLIER, Florent. Des fruits et des hommes. L'arboriculture fruitière en Ile-de-France (vers 1600-vers 1800). Rennes: Presses Universitaires de Rennes, 2003). Les traités de culture des arbres fruitiers ont toujours un chapitre sur la confiture.

12. - Le 9 mai 1748, Nicolas Petitbon est « reçu et installé....pour le bon plaisir de monsieur le comte de Caraman seigneur de ce lieu dans l'état et office de garde messier des avenues tant fruitières que autre dans l'étendue de la seigneurie de ce lieu ». Archives dép. du Val-d'Oise, B95/2847. Mention extraite de 
QUELLIER, Florent. Des fruits et des hommes. L'arboriculture fruitière en Ile-de-France (vers 1600-vers 1800). Rennes : Presses Universitaires de Rennes, 2003, p. 154, note 157.

13. - Blondel, 1737, vol. I, p. 80.

14. - SCHABOL, Jean-Roger. Discours historiques sur le village de Montreuil. Réédition de la Société Régionale d'Horticulture de Montreuil, 2007, p. 21.

15. - HIVERT, Philippe. Montreuil-sous-bois, Tome 3, D'un empire à l'autre (1795-1871). Eden/Folies d'Encre, 2003, p. 132.

16. - LANGLOIS, Hyppolite. Le livre de Montreuil-aux-pêches, Théorie et pratique de la culture de ses arbres, avec la collaboration des principaux arboriculteurs du pays. Paris : Librairie Firmin-Didot frères, fils et Compagnie, 1875, p. 99.

17. - COURELIS, Thalie. Les horticulteurs de Montreuil : logement et usine. Mémoire de Master 2, Archéologie moderne et contemporaine, Université de Paris IV-La Sorbonne, 2009. 2 vol., 93 et 88 pages.

18. - Les maisons sont situées aux 8 et 52 rue de Romainville, aux 77 et 79 rue Danton, au 68 rue de Rosny, 29 rue Savart et encore au 61 rue Victor Hugo.

19. - Une inscription sur la façade de la maison mentionne le nom de l'architecte ainsi que la date de construction.

20. - Archives municipales de Montreuil.

21. - Pour emprunter l'expression à Gilbert Simondon.

22. - À quelque biais près cette répartition semble plausible; elle appelle bien entendu une sévère critique des sources qu'il n'était pas possible de mener dans le cadre de cette petite note.

23. - $\mathrm{AD} / 77-\mathrm{a}-/ 2 \mathrm{BP} / 1633$.

24. - $\mathrm{AD} / 77-\mathrm{a}-/ 2 \mathrm{BP} / 3700$.

25. - Arch. Dép. de la Seine-Saint-Denis-/2E131/244.

26. - Bien entendu nos 129 stockeurs imprécis ne sauraient être ignorés et il m’a semblé qu'on pouvait les retrouver au moment de comparer notre modèle de localisation aux caractéristiques des stocks fruitiers eux-mêmes définis en termes de nature des fruits et modes de stockages.

27. - AD/93-h-/2E131/127 : dans le cellier : « ...une hotte dosier servant à porter vendre, un hotereau un noguet ou panier a fromage... ».

28. - $\mathrm{AD} / 77-\mathrm{C}-/ \mathrm{B} / 414, \mathrm{IAD}$ du 24-11-1759, à la requête de Jeanne Debuis, sa femme.

29. - PÉRU, Jean-Jacques. "Pomologues, chemins de fer et agronomes d'Etat: les luttes pour le contrôle variétal des poires et des pommes de "marché » en France de 1828 à 1937 ». Le patrimoine fruitier, actes du Colloque de l'AFCEV de la Ferté-Bernard d'octobre 1998, éd. INRA.

\section{RÉSUMÉS}

Le fruit est le dessert par excellence à l'époque moderne. Le fruitier ou fruiterie, entendu comme lieu de mûrissement et de stockage des fruits, est peu connu en Île-de-France, région longtemps (et encore) productrice de fruits pour Paris et le plus grand centre de consommation de l'Occident médiéval et moderne. Outre une ceinture discontinue de jardins et de vergers autour 
de Paris, la vallée de la Seine à l'ouest et les terroirs proches de Versailles, la vallée de Montmorency, les secteurs de Montreuil et la région de Corbeil et Thomery fournissent l'essentiel de la production locale. Dans cet article, nous tenterons de faire le point sur les connaissances agronomiques ou "théoriques" des anciens sur les conditions nécessaires à ce lieu de stockage, puis nous confronterons cette connaissance livresque à trois types d'observations plus empiriques: les vestiges interprétés d'un fruitier lors d'une fouille archéologique, les observations ethnographiques effectuées sur quelques maisons d'horticulteurs de Montreuil, haut lieu de l'arboriculture fruitière francilienne, et enfin le mode de stockage des fruits tel qu'il apparaît dans les archives du monde paysan d'île-de-France aux XVIIIe et XIXe siècles.

INDEX

Mots-clés : stockage, fruits, fruitier, Montreuil, archéologie, archives, Île-de-France

\section{AUTEURS}

\section{JEAN-YVES DUFOUR}

Archéologue, Institut national de recherches archéologiques préventives, UMR 7041 équipe

Archéologies environnementales Jean-yves.dufour@inrap

\section{THALIE COURELIS}

Diplômée d'un Master d'Archéologie Moderne et Contemporaine à l'Université Paris IV - La Sorbonne en 2009 courelisthalie@yahoo.fr

\section{JEAN-JACQUES PÉRU}

Conservateur du patrimoine, actuellement rattaché au CNFPT jean-jacques.peru@wanadoo.fr 